\title{
Coeficiente de difusão efetivo e modelagem matemática da secagem de sementes de crambe
}

\author{
Lílian M. Costa $^{1}$, O svaldo Resende ${ }^{1}$, Kelly A. Sousa ${ }^{1} \&$ Douglas N. Gonçalves ${ }^{1}$
}

\section{RESU M O}

O mecanismo através do qual ocorre a difusão da água nos produtos agrícolas, é complexo devido à diversidade da composição química e estrutura física dos produtos. 0 objetivo no presente trabalho foi ajustar diversos modelos matemáticos ao processo de secagem das sementes de crambe (Crambe abyssinica), em diferentes condições de ar, determinar o coeficiente de difusão efetivo e obter a energia de ativação. As sementes de crambe utilizadas foram colhidas com teor de água de 0,26 (decimal b.s.) e secadas até 0 teor de 0,09 (decimal b.s.). A secagem do produto foi realizada sob variadas condições controladas nas temperatura de $30,40,50,60$ e $70{ }^{\circ} \mathrm{C}$ e umidades relativas de 37,$4 ; 22,3 ; 14,0 ; 8,3$ e $5,1 \%$, respectivamente. Dentre os modelos que apresentaram melhor ajuste aos dados experimentais, 0 de $W$ ang e Singh foi o que melhor se ajustou aos dados nas diferentes condições de secagem. 0 coeficiente de difusão efetivo aumentou com a elevação da temperatura apresentando valores de 0,88 $\times 10^{-11} ; 1,99 \times 10^{-11} ; 2,90 \times 10^{-11} ; 3,48 \times 10^{-11}$ e $5,85 \times 10^{-11} \mathrm{~m}^{2} \mathrm{~s}^{-1}$, para as temperaturas de $30,40,50,60$ e $70^{\circ} \mathrm{C}$, respectivamente, durante a secagem do crambe. A relação entre o coeficiente de difusão efetivo e a temperatura de secagem pode ser descrita pela equação de Arrhenius, que apresenta uma energia de ativação para a difusão líquida na secagem de $37,07 \mathrm{~kJ} \mathrm{~mol}^{-1}$.

Palavras-chave: difusão líquida, movimentação de água, oleaginosa

\section{Effective diffusion coefficient and mathematical modeling for drying of crambe seeds}

\begin{abstract}
The mechanism of the water diffusion in agricultural products is complex due to the diversity of chemical composition and physical structure of the products. The aim of this work was to fit different mathematical models to the drying process of the crambe seeds (Crambe abyssinica), under varying conditions of air and to determine the effective diffusion coefficient and obtain the activation energy. Crambe seeds harvested with moisture content of 0.26 (decimal d.b.) and dried until 0.09 (decimal d.b.). D rying was carried out under different conditions of controlled temperature $30,40,50,60$ and $70^{\circ} \mathrm{C}$ and relative humidity of $37.4 ; 22.3 ; 14.0 ; 8.3$ and $5.1 \%$, respectively. Among the models that presented the best fit for the experimental data, Wang e Singh was the best fit to the data at different drying conditions. The effective diffusion coefficient increased with increasing temperature with values of $0.88 \times 10^{-11} ; 1.99 \times$ $10^{-11} ; 2.90 \times 10^{-11} ; 3.48 \times 10^{-11}$ and $5.85 \times 10^{-11} \mathrm{~m}^{2} \mathrm{~s}^{-1}$, for temperatures of $30,40,50,60$ e $70^{\circ} \mathrm{C}$, respectively, during crambe drying. The relationship between the effective diffusion coefficient and drying temperature can be described by the Arrhenius equation, which presents an activation energy for liquid diffusion in the drying of $37.07 \mathrm{~kJ} \mathrm{~mol}^{-1}$.
\end{abstract}

Key words: liquid diffusion, water movement, oilseed 


\section{INTRODUÇÃO}

O crambe (Crambe abyssinica) é uma planta da família das brassicaceae e surge como alternativa para a produção de matéria-prima visando à obtenção de biocombustível. Depois da colheita o crambe deve ser submetido a secagem para reduzir seu teor de água até 0,11 (decimal b.s.) e garantir o armazenamento seguro do produto (Oplinger et al., 2000). De acordo com Golz (1993), a temperatura de secagem quanto à manutenção da qualidade das sementes do crambe deve ser, no máximo, de $43,3^{\circ} \mathrm{C}$.

Estudos sobre sistemas de secagem, seu dimensionamento, otimização e a determinação da viabilidade de sua aplicação comercial, podem ser feitos por simulação matemática. Para a simulação, cujo princípio se fundamenta na secagem de sucessivas camadas delgadas do produto, utiliza-se um modelo matemático que representa satisfatoriamente a perda de umidade do produto durante o período de secagem. Na literatura se encontram vários métodos propostos para se analisar a secagem em camada fina de produtos higroscópicos: os teóricos, os semiteóricos e os empíricos (Afonso Júnior \& Corrêa, 1999).

As curvas de secagem em camada delgada variam com a espécie, variedade, condições ambientais e métodos de preparo pós-colheita, entre outros fatores. Neste sentido, diversos modelos matemáticos têm sido utilizados para descrever o processo de secagem de produtos agrícolas (Resende et al., 2008; 2009; 2010). Segundo Brooker et al. (1992), a maioria dos modelos empregados para representar a secagem dos produtos agrícolas foi desenvolvida negligenciando-se a importância da contração volumétrica do produto durante o processo de desidratação. A representação matemática do processo de secagem de diversos produtos agrícolas vem, já há algum tempo, sendo estudada e utilizada na tentativa de predizer os fenômenos que ocorreram durante este processo. Inúmeros modelos matemáticos têm sido estudados com o objetivo de projetar, controlar e otimizar unidades de secagem (Rocha et al., 2005).

Diversas teorias e fórmulas empíricas foram desenvolvidas para predizer a taxa de secagem: Teoria da difusão líquida, Teoria capilar, Teoria de Luikov, Teoria de Philip e de Vries, Teoria de Krisher-Berger e Pei e Teoria da condensação-evaporação. As duas primeiras são básicas e fundamentam as outras (Martinazzo et al., 2007). Atualmente, pesquisas relacionadas ao estudo da cinética de secagem em camada delgada são realizadas com diversos produtos agrícolas, como sementes, grãos, frutos e em algumas espécies de plantas, com importância econômica. Observa-se, conforme o produto e as condições do processo de secagem, que diferentes modelos se ajustam a cada situação específica (Martinazzo et al., 2007)

Segundo Brooker et al. (1992), na teoria da difusão líquida a segunda lei de Fick tem sido utilizada para estabelecer a difusão da água em função do gradiente de concentração. O coeficiente de difusão é uma difusividade efetiva que engloba os efeitos de todos os fenômenos, podendo intervir sobre a migração da água e o seu valor é sempre obtido pelo ajuste das curvas experimentais (Martinazzo et al., 2007). A solução da equação de difusão utilizada é uma das mais simples e parece ser a principal razão de seu emprego. Pode-se entender a difusividade como a facilidade com que a água é removida do material. Como a difusividade varia conforme mudam as condições de secagem (temperatura e velocidade do ar), ela não éintrínseca ao material. Convenciona-se chamá-la, assim, de difusividade efetiva (Oliveira et al., 2006).

Objetivou-se, no presente trabalho, ajustar modelos matemáticos ao processo de secagem das sementes de crambe (Crambe abyssinica) em diversas condições de ar, determinar o coeficiente de difusão efetivo e obter a energia de ativação para o processo.

\section{Material e MÉTODOS}

Este trabalho foi realizado no Laboratório de Pós-colheita de Produtos Vegetais do Instituto Federal de Educação, Ciência e Tecnologia Goiano - Campus Rio Verde, Goiás, Brasil (IFGoiano-Campus Rio Verde). A colheita do crambe (Crambe abissynica) da cultivar FMS Brilhante foi realizada manualmente, com o teor de água das sementes de 0,26 (decimal b.s.). A secagem foi realizada em estufa Marconi® MA-035, com ventilação forçada, nas seguintes condições de ar: temperaturas de secagem de $30,40,50,60$ e $70{ }^{\circ} \mathrm{C}$ e umidades relativas de 37,$4 ; 22,3 ; 14,0 ; 8,3$ e $5,1 \%$, respectivamente.

A temperatura e a umidade relativa do ar de secagem foram monitoradas por meio de um psicrômetro instalado no interior do secador. Durante o processo de secagem as bandejas com $400 \mathrm{~g}$ das amostras com quatro repetições por temperatura, foram pesadas periodicamente, até o teor de água de 0,09 (decimal b.s).

Os teores de água do produto foram determinados por gravimetria utilizando-se estufa a $105 \pm 1{ }^{\circ} \mathrm{C}$, durante $24 \mathrm{~h}$, em três repetições (Brasil, 2009).

Para obtenção do equilíbrio higroscópico do crambe foram utilizadas três repetições, contendo $10 \mathrm{~g}$ de produto, mantidas nas mesmas condições de secagem e pesadas periodicamente, até a massa permanecer constante. As razões de umidade do produto foram determinadas pela expressão:

$$
\mathrm{RU}=\frac{\mathrm{X}^{*}-\mathrm{X}_{\mathrm{e}}^{*}}{\mathrm{X}_{\mathrm{i}}^{*}-\mathrm{X}_{\mathrm{e}}^{*}}
$$

em que:

RU - razão de umidade do produto, adimensional

$\mathrm{X}^{*}$ - teor de água do produto, decimal (b.s.)

$\mathrm{X}^{*}$ - teor de água de equilíbrio do produto, decimal (b.s.)

$\mathrm{X}_{\mathrm{i}}{ }^{\mathrm{e}}$ - - teor de água inicial do produto, decimal (b.s.)

Aos dados experimentais de secagem do crambe foram ajustados os modelos matemáticos frequentemente utilizados para representação da secagem de produtos agrícolas (Afonso Júnior \& Corrêa, 1999; Akpinar et al., 2003; Ertekin \& Yaldiz, 2004; Lahsasni et al., 2004), cujas expressões estão apresentadas na Tabela 1. 
Tabela 1. M odelos matemáticos utilizados para predizer o fenômeno de secagem de produtos agrícolas

\begin{tabular}{lcr}
\hline Designação do modelo & Modelo & (2) \\
Werma e Singh & $\mathrm{RU}=1+\mathrm{at}+\mathrm{bt}^{2}$ & $(3)$ \\
Thompson & $\mathrm{RU}=\mathrm{a} \exp (-\mathrm{kt})+(1-\mathrm{a}) \exp \left(-\mathrm{k}_{1} \mathrm{t}\right)$ & $(4)$ \\
Page & $\mathrm{RU}=\exp \left\{\left[-\mathrm{a}-\left(\mathrm{a}^{2}+4 \mathrm{bt}\right)^{0,5}\right] / 2 \mathrm{~b}\right\}$ & $(5)$ \\
Newton & $\mathrm{RU}=\exp \left(-\mathrm{kt} \mathrm{t}^{\mathrm{n}}\right)$ & $(6)$ \\
Midilli & $\mathrm{RU}=\exp (-\mathrm{kt})$ & $(7)$ \\
Logarítmico & $\mathrm{RU}=\mathrm{a} \exp \left(-\mathrm{kt} \mathrm{t}^{\mathrm{n}}\right)+\mathrm{bt}$ & $(8)$ \\
Henderson e Pabis & $\mathrm{RU}=\mathrm{a} \exp (-\mathrm{kt})+\mathrm{c}$ & $(19)$ \\
Henderson e Pabis Mod. & $\mathrm{RU}=\mathrm{a} \exp (-\mathrm{kt})$ & $(11)$ \\
Exponencial de Dois Termos & $\mathrm{RU}=\mathrm{a} \exp (-\mathrm{kt})+\mathrm{b} \exp (-\mathrm{k} \mathrm{t} \mathrm{t})+\mathrm{cexp}\left(-\mathrm{k}_{1} \mathrm{t}\right)$ & $(12)$ \\
Dois Termos & $\mathrm{RU}=\mathrm{a} \exp (-\mathrm{kt})+(1-\mathrm{a}) \exp (-\mathrm{kat})$ & $(13)$ \\
Aproximação da Difusão & $\mathrm{RU}=\mathrm{a} \exp \left(-\mathrm{k}_{\mathrm{o}} \mathrm{t}\right)+\mathrm{b} \exp \left(-\mathrm{k}_{1} \mathrm{t}\right)$ & $\mathrm{RU}=\mathrm{a} \exp (-\mathrm{kt})+(1-\mathrm{a}) \exp (-\mathrm{kbt})$ \\
\hline
\end{tabular}

em que:

$\mathrm{k}, \mathrm{k}_{\mathrm{o}}, \mathrm{k}_{1}$ - constantes de secagem, $\mathrm{s}^{-1}$

$\mathrm{a}, \mathrm{b}, \mathrm{c}, \mathrm{n}$ - coeficientes dos modelos

Para o ajuste dos modelos foram realizadas análises de regressão não linear, pelo método Gauss-Newton, utilizandose um programa estatístico, o erro médio estimado (SE), a magnitude do erro médio relativo (P), a magnitude do coeficiente de determinação - $\mathrm{R}^{2}$ (que, apesar de não ser um bom parâmetro para verificar o ajuste de modelos não-lineares, normalmente apresenta maiores valores para os modelos que melhor se ajustam aos dados experimentais), e a verificação do comportamento da distribuição dos resíduos. Os erros médios relativo e estimado para cada um dos modelos foram calculados conforme as seguintes expressões:

$$
\begin{aligned}
& \mathrm{SE}=\sqrt{\frac{\sum(\mathrm{Y}-\hat{\mathrm{Y}})^{2}}{\mathrm{GLR}}} \\
& \mathrm{P}=\frac{100}{\mathrm{n}} \sum \frac{|\mathrm{Y}-\hat{\mathrm{Y}}|}{\mathrm{Y}}
\end{aligned}
$$

em que:

Y - valor observado experimentalmente

$\mathrm{v}$ - valor estimado pelo modelo

n - número de observações experimentais

O modelo matemático da difusão líquida com a solução analítica para a forma geométrica esférica, com aproximação de oito termos (Eq. 16), foi ajustado aos dados experimentais de secagem do crambe, considerando-se o raio equivalente inicial dos grãos, mantendo-o constante ao longo da secagem.

$$
\mathrm{RU}=\frac{\mathrm{X}^{*}-\mathrm{X}_{\mathrm{e}}^{*}}{\mathrm{X}_{\mathrm{i}}^{*}-\mathrm{X}_{\mathrm{e}}^{*}}=\frac{6}{\pi^{2}} \sum_{\mathrm{n}=1}^{\infty} \frac{1}{\mathrm{n}^{2}} \exp \left[-\frac{\mathrm{n}^{2} \pi^{2} \mathrm{Dt}}{9}\left(\frac{3}{\mathrm{R}}\right)^{2}\right]
$$

em que:

$$
\begin{aligned}
& \text { RU - razão de umidade, adimensional } \\
& \text { D - coeficiente de difusão efetivo, } \mathrm{m}^{2} \mathrm{~s}^{-1} \\
& \text { n - número de termos } \\
& R \text { - raio de esfera equivalente } 0,0037 \mathrm{~m} \\
& \mathrm{~T} \text { - tempo, } \mathrm{s}
\end{aligned}
$$

O raio equivalente é definido como o raio de uma esfera com volume equivalente ao do grão. Obteve-se o volume de cada grão $\left(\mathrm{V}_{\mathrm{g}}\right)$ por meio da medição dos três eixos ortogonais (comprimento, largura e espessura), em trinta grãos, no início da secagem, com auxílio de um paquímetro digital modelo 176BL com resolução de $0,01 \mathrm{~mm}$, de acordo com a expressão proposta por Mohsenin (1986):

$$
\mathrm{Vg}=\frac{\pi(\mathrm{a} \cdot \mathrm{b} \cdot \mathrm{c})}{6}
$$

em que:

$$
\begin{array}{ll}
\mathrm{V}_{\mathrm{g}} & \text { - volume do grão, } \mathrm{m}^{3} \\
\mathrm{~A} & \text { - maior eixo do grão, } \mathrm{m} \\
\mathrm{B} & \text { - eixo médio do grão, } \mathrm{m} \\
\mathrm{C} & \text { - menor eixo do grão, } \mathrm{m}
\end{array}
$$

A relação do aumento do coeficiente de difusão com a elevação da temperatura do ar de secagem foi descrita por meio da equação de Arrhenius.

$$
\mathrm{D}=\mathrm{D}_{\mathrm{o}} \exp \left(\frac{-\mathrm{E}_{\mathrm{a}}}{\mathrm{R} \cdot \mathrm{T}_{\mathrm{a}}}\right)
$$

em que:

$$
\begin{array}{ll}
\mathrm{D}_{\mathrm{o}} & \text { - fator pré-exponencial } \\
\mathrm{E}_{\mathrm{a}} & \text { - energia de ativação, } \mathrm{kJ} \mathrm{mol}^{-1} \\
\mathrm{R}^{-1} & \text { - constante universal dos gases, } 8,134 \mathrm{~kJ} \mathrm{kmol}^{-1} \cdot \mathrm{K}^{-1} \\
\mathrm{~T}_{\mathrm{a}} & \text { - temperatura absoluta, } \mathrm{K}
\end{array}
$$


Os coeficientes da expressão de Arrhenius foram obtidos linearizando-se a Eq. 19 com a aplicação do logaritmo, da seguinte forma:

$$
\mathrm{LnD}=\mathrm{LnD}_{\mathrm{o}}-\frac{\mathrm{E}_{\mathrm{a}}}{\mathrm{R}} \cdot \frac{1}{\mathrm{~T}_{\mathrm{a}}}
$$

Os valores do coeficiente de difusão efetivo foram submetidos à análise de variância e ao teste de comparação de médias de Tukey a $5 \%$ de probabilidade.

\section{RESULTADOS E DISCUSSÃO}

Na Figura 1 se apresentam as curvas de secagem em diversas condições de ar para o Crambe abyssinica.

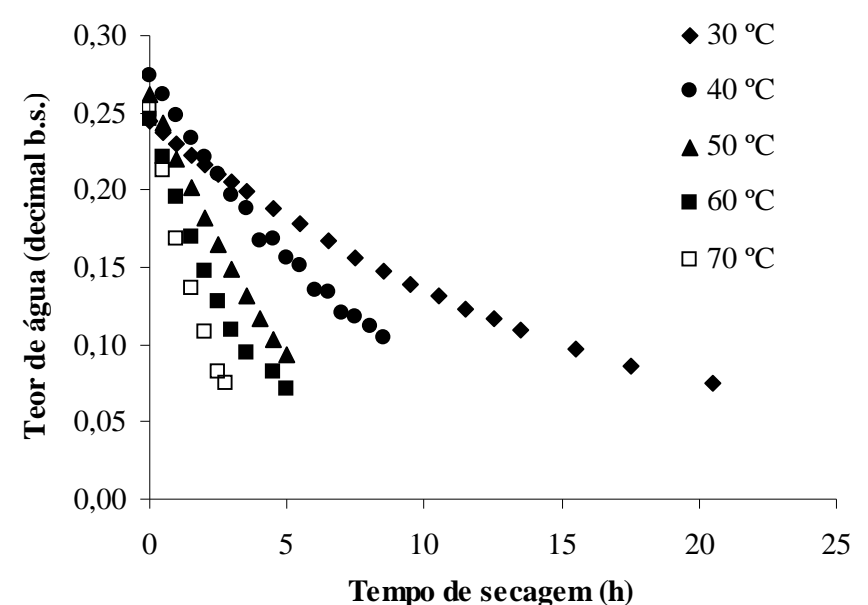

Figura 1. Curvas de secagem das sementes de Crambe abyssinica nas temperaturas de $30,40,50,60$ e $70^{\circ} \mathrm{C}$

Verificou-se que os tempos de secagem das sementes de crambe, considerando-se a redução do teor de água de 0,26 para 0,09 (decimal b.s.), nas temperaturas de 30, 40, 50, 60 e 70 ${ }^{\circ} \mathrm{C}$ foram de 20,$5 ; 8,5 ; 5,0 ; 5.0$ e $2,75 \mathrm{~h}$, respectivamente e que, com o aumento da temperatura, ocorreu diminuição do tempo de secagem evidenciando a maior velocidade de retirada de água. Pode-se observar que a cinética de secagem do crambe apresenta as mesmas características da maioria dos grãos agrícolas (Almeida et al., 2009) ou seja, existe uma clara dependência da velocidade da secagem com a temperatura do ar. Segundo Peske et al. (2003), a utilização de temperaturas elevadas permite uma secagem mais rápida porém pode provocar diferença de teor de água entre a periferia e o centro da semente, gerando um gradiente de pressão possível de causar danificação mecânica.

Na Tabela 2 se apresenta o resumo do ajuste dos doze modelos analisados por meio de regressãonão linear aos dados experimentais de secagem de crambe em camada fina, considerando-se as diferentes temperaturas do ar de secagem.

O modelo Wang e Singh apresentou menor valor de erro médio estimado (SE) e magnitude do erro médio relativo (P) inferior a $10 \%$ indicando, de acordo com Mohapatra \& Rao
(2005), ser adequado para a descrição do processo. Em todas as condições de secagem o valor mais elevado do coeficiente de determinação $\left(\mathrm{R}^{2}\right)$, foi superior a 0,98 sinalizando, de acordo com Madamba et al. (1996), uma representação satisfatória do processo de secagem. Com relação à distribuição de resíduos, este modelo foi aleatório quando ajustado aos dados de secagem a 30,40 e $50^{\circ} \mathrm{C}$, prevendo melhores ajustes aos dados experimentais. Verifica-se, ainda, que o modelo Midilli também se ajustou satisfatoriamente aos dados de secagem a 50 e $60^{\circ} \mathrm{C}$ enquanto o Logarítmo aos de 60 e $70^{\circ} \mathrm{C}$ e o de Aproximação da Difusão também se ajustou satisfatoriamente aos dados de secagem a $30^{\circ} \mathrm{C}$. Dentre os modelos que apresentaram melhor ajuste aos dados experimentais, o modelo Wang e Singh foi selecionado por se ajustar adequadamente aos dados nas diferentes condições de secagem do crambe, conforme ilustrado na Figura 2. Doymaz (2004) observou que os modelos Exponencial de dois termos e o de Wang e Singh foram os que melhor representaram os dados experimentais da secagem de ameixa.

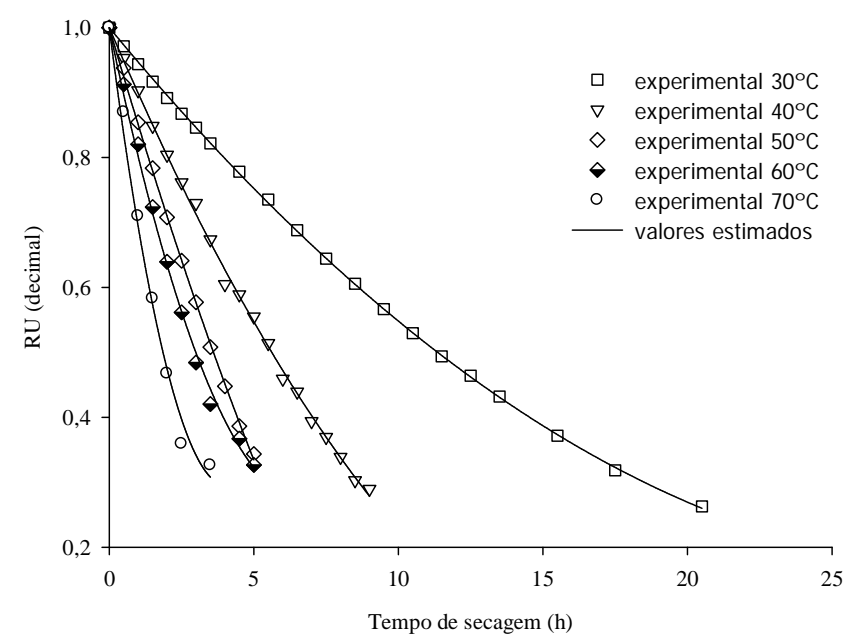

Figura 2. Valores da cinética de secagem das sementes de Crambe abyssinica, experimentais e estimados pelo modelo de W ang Singh, para diversas condições de secagem

Na Tabela 3 se acham os coeficientes do modelo de Wang Singh ajustados aos dados experimentais da cinética de secagem do crambe, nas diferentes condições de temperatura.

Analisando os resultados apresentados na Tabela 3 tem-se que as magnitudes dos coeficientes "a" e "b" para o modelo de Wang Singh, variam quadraticamente com a elevação da temperatura do ar de secagem. Desta forma, a cinética de secagem do crambe pode ser estimada para a faixa de temperatura estudada, utilizando-se a seguinte expressão:

$$
\begin{aligned}
\mathrm{RU} & =1+\left(-0,0001 \cdot \mathrm{T}^{2}+0,0061 \cdot \mathrm{T}-0,1256\right) \cdot \mathrm{t}+ \\
& +\left(0,00004 \cdot \mathrm{T}^{2}-0,0034 \cdot \mathrm{T}+0,066\right) \cdot \mathrm{t}^{2}
\end{aligned}
$$

em que:

$\mathrm{T}$ - temperatura de secagem, ${ }^{\circ} \mathrm{C}$

t - tempo de secagem, h 
Tabela 2. Erro médio estimado ( $S E$, decimal), erro médio relativo $(P, \%)$, coeficiente de determinação ( $\left.{ }^{2}, \%\right)$ e tendência de distribuição dos resíduos (A-al eatória; $T$-tendenciosa) durante a secagem do crambe nas diversas condições de temperatura

\begin{tabular}{|c|c|c|c|c|c|c|c|c|}
\hline \multirow{2}{*}{ Modelos } & \multirow{2}{*}{$\begin{array}{c}\text { SE } \\
\text { (decimal) }\end{array}$} & $\mathbf{P}$ & $\mathbf{R}^{2}$ & \multirow{2}{*}{$\begin{array}{l}\text { Distribuição } \\
\text { dos Resíduos }\end{array}$} & \multirow{2}{*}{$\begin{array}{c}\text { SE } \\
\text { (decimal) }\end{array}$} & $\mathbf{P}$ & $\mathbf{R}^{2}$ & \multirow{2}{*}{$\begin{array}{l}\text { Distribuição } \\
\text { dos Resíduos }\end{array}$} \\
\hline & & \multicolumn{2}{|c|}{$(\%)$} & & & \multicolumn{2}{|c|}{$(\%)$} & \\
\hline & \multicolumn{4}{|c|}{$30^{\circ} \mathrm{C}$} & \multicolumn{4}{|c|}{$40^{\circ} \mathrm{C}$} \\
\hline Wang e Singh & 0,003 & 0,36 & 99,99 & A & 0,008 & 1,17 & 99,92 & $A$ \\
\hline Verma & 0,149 & 23,64 & 78,47 & $T$ & 0,076 & 13,64 & 94,89 & $T$ \\
\hline Thompson & 0,013 & 2,02 & 99,83 & $T$ & 0,025 & 4,23 & 99,40 & $\mathrm{~T}$ \\
\hline Page & 0,005 & 0,72 & 99,97 & $T$ & 0,010 & 1,44 & 99,90 & $T$ \\
\hline Newton & 0,013 & 2,02 & 99,84 & $T$ & 0,024 & 4,23 & 99,40 & $T$ \\
\hline Midilli & 0,004 & 0,49 & 99,98 & $T$ & 0,009 & 1,18 & 99,93 & $T$ \\
\hline Logarítmico & 0,004 & 0,64 & 99,98 & $T$ & 0,009 & 1,21 & 99,92 & $\mathrm{~T}$ \\
\hline Henderson e Pabis & 0,011 & 1,67 & 99,88 & A & 0,020 & 3,28 & 99,61 & $\mathrm{~T}$ \\
\hline Henders on e Pabis Modificado & 0,003 & 0,43 & 99,99 & $T$ & 0,010 & 1,22 & 99,92 & $\mathrm{~T}$ \\
\hline Exponencial de Dois Termos & 0,013 & 2,02 & 99,84 & $T$ & 0,025 & 4,23 & 99,40 & $\mathrm{~T}$ \\
\hline Dois Termos & 0,012 & 1,67 & 99,88 & $T$ & 0,009 & 1,20 & 99,92 & $\mathrm{~T}$ \\
\hline \multirow[t]{2}{*}{ Aproximação da Difusão } & 0,003 & 0,44 & 99,98 & A & 0,009 & 1,22 & 99,92 & $A$ \\
\hline & \multicolumn{4}{|c|}{$50^{\circ} \mathrm{C}$} & \multicolumn{4}{|c|}{$60 \div \mathrm{C}$} \\
\hline Wang e Singh & 0,006 & 0,80 & 99,96 & A & 0,016 & 2,30 & 99,76 & $T$ \\
\hline Verma & 0,189 & 28,42 & 65,05 & $T$ & 0,240 & 39,68 & 95,96 & $\mathrm{~T}$ \\
\hline Thompson & 0,029 & 4,11 & 99,26 & $T$ & 0,019 & 2,62 & 99,69 & $\mathrm{~T}$ \\
\hline Page & 0,006 & 0,71 & 99,97 & $T$ & 0,018 & 2,78 & 99,72 & $\mathrm{~T}$ \\
\hline Newton & 0,027 & 4,11 & 99,26 & $T$ & 0,018 & 2,62 & 99,69 & $\mathrm{~T}$ \\
\hline Midilli & 0,003 & 0,50 & 99,98 & A & 0,012 & 1,85 & 99,84 & $\mathrm{~T}$ \\
\hline Logarítmico & 0,006 & 0,75 & 99,97 & A & 0,018 & 2,51 & 99,73 & $\mathrm{~T}$ \\
\hline Henderson e Pabis & 0,025 & 3,12 & 99,54 & $T$ & 0,020 & 2,67 & 99,72 & $\mathrm{~T}$ \\
\hline Henderson e Pabis Modificado & 0,005 & 0,60 & 99,97 & $T$ & 0,018 & 2,42 & 99,74 & $\mathrm{~T}$ \\
\hline Exponencial de Dois Termos & 0,005 & 0,67 & 99,97 & $T$ & 0,019 & 2,62 & 99,69 & $\mathrm{~T}$ \\
\hline Dois Termos & 0,005 & 0,60 & 99,98 & $T$ & 0,017 & 2,67 & 99,72 & $\mathrm{~T}$ \\
\hline \multirow[t]{2}{*}{ Aproximaç̧ão da Difusão } & 0,038 & 4,11 & 99,26 & $T$ & 0,025 & 2,71 & 99,69 & $\mathrm{~T}$ \\
\hline & \multicolumn{4}{|c|}{$700 \mathrm{C}$} & & & & \\
\hline Wang e Singh & 0,025 & 3,63 & 99,57 & $T$ & & & & \\
\hline Verma & 0,029 & 3,05 & 99,55 & $\mathrm{~T}$ & & & & \\
\hline Thompson & 0,033 & 5,14 & 99,26 & $T$ & & & & \\
\hline Page & 0,033 & 5,15 & 99,28 & $T$ & & & & \\
\hline Newton & 0,030 & 5,15 & 99,26 & $T$ & & & & \\
\hline Midilli & 0,012 & 2,16 & 99,87 & $T$ & & & & \\
\hline Logarítmico & 0,035 & 4,92 & 99,36 & $T$ & & & & \\
\hline Henders on e Pabis & 0,042 & 5,18 & 99,30 & $T$ & & & & \\
\hline Henders on e Pabis Modificado & 0,007 & 0,71 & 99,97 & A & & & & \\
\hline Exponencial de Dois Termos & 0,033 & 5,13 & 99,27 & $T$ & & & & \\
\hline Dois Termos & 0,022 & 2,58 & 99,68 & $\mathrm{~T}$ & & & & \\
\hline
\end{tabular}

Tabela 3. Coeficientes do modelo de W ang Singh ajustados para a secagem do crambe em diferentes condições de temperatura e umidade relativa do ar com as respectivas equações

\begin{tabular}{ccccccc}
\hline \multirow{2}{*}{ Coeficientes } & \multicolumn{5}{c}{ Temperatura $\left({ }^{\circ} \mathbf{C}\right)$} & \\
\cline { 2 - 6 } & $\mathbf{3 0}$ & $\mathbf{4 0}$ & $\mathbf{5 0}$ & $\mathbf{6 0}$ & $\mathbf{7 0}$ & Equação \\
$\mathrm{a}$ & $-0,053849$ & $-0,103624$ & $-0,153214$ & $-0,213054$ & $-0,349369$ & $\mathrm{a}=-0,0001 T^{2}+0,0061 \mathrm{-}-0,1256$ \\
$\mathrm{~b}$ & 0,000866 & 0,002611 & 0,004024 & 0,015801 & 0,043316 & $\mathrm{~b}=0,00004 T^{2}-0,0034 \mathrm{~T}+0,066$ \\
\hline
\end{tabular}

Encontram-se, na Figura 3, os dados experimentais e calculados de razão de umidade (RU) empregando-se o modelo de Wang Singh com os parâmetros estimados por meio da Eq. 20.

A Figura 3 possibilita verificar o ajuste deste modelo na descrição da secagem do crambe. Observa-se uma superestimação da razão de umidade para a maioria dos valores experimentais; entretanto, com a redução dos valores da razão de umidade ocorre uma discrepância maior entre os dados experimentais e os estimados pelo modelo.

Na Figura 4 estão os valores do coeficiente de difusão efetivo para as sementes de Crambe abyssinica durante as diferentes condições de secagen.
Verifica-se que durante a secagem do crambe o coeficiente de difusão efetivo aumenta significativamente $(\mathrm{P}<0,05)$, com a elevação da temperatura, apresentando valores de $0,88 \times 10^{-11}$; $1,99 \times 10^{-11} ; 2,90 \times 10^{-11} ; 3,48 \times 10^{-11}$ e $5,85 \times 10^{-11} \mathrm{~m}^{2} \mathrm{~s}^{-1}$ para as temperaturas de $30,40,50,60$ e $70^{\circ} \mathrm{C}$, respectivamente.

Goneli (2008), estudando a secagem de sementes de mamona, encontrou magnitudes de coeficientes de difusão efetivo entre $0,5117 \times 10^{-10} \mathrm{e} 1,5643 \times 10^{-10} \mathrm{~m}^{2} \mathrm{~s}^{-1}$ para a faixa de temperatura de 25 a $55{ }^{\circ} \mathrm{C}$. Almeida et al. (2009), verificaram que durante a secagem do feijão adzuki os coeficientes de difusão efetivo apresentaram magnitudes entre $0,51 \times 10^{-10} \mathrm{e} 2,23 \times 10^{-10} \mathrm{~m}^{2} \mathrm{~s}^{-1}$ para a faixa de temperatura de 30 a $70{ }^{\circ} \mathrm{C}$; o mesmo ocorreu para 


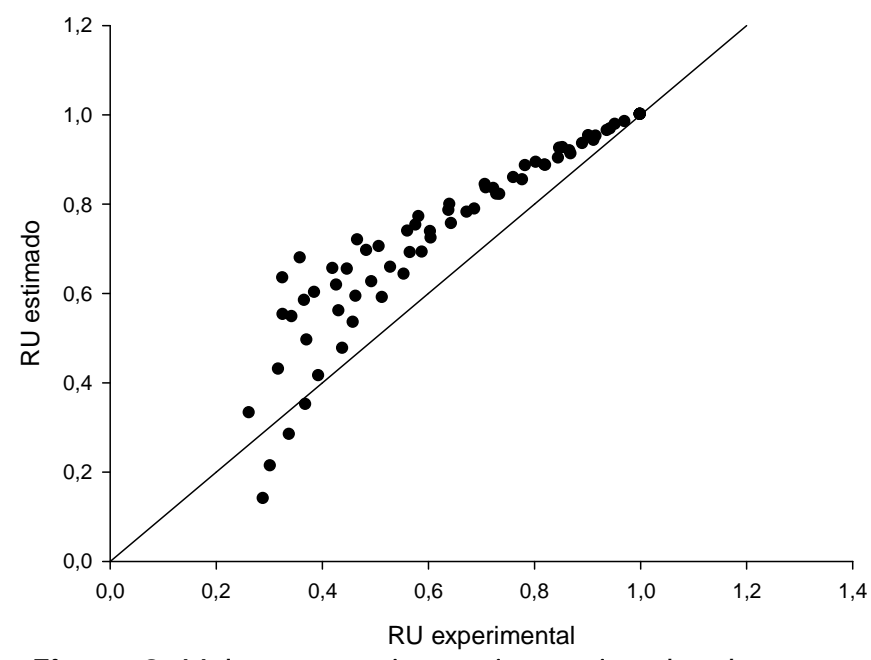

Figura 3. Valores experimentais e estimados de razão de umidade, obtidos pelo modelo de W ang Singh em função da temperatura de secagem do crambe

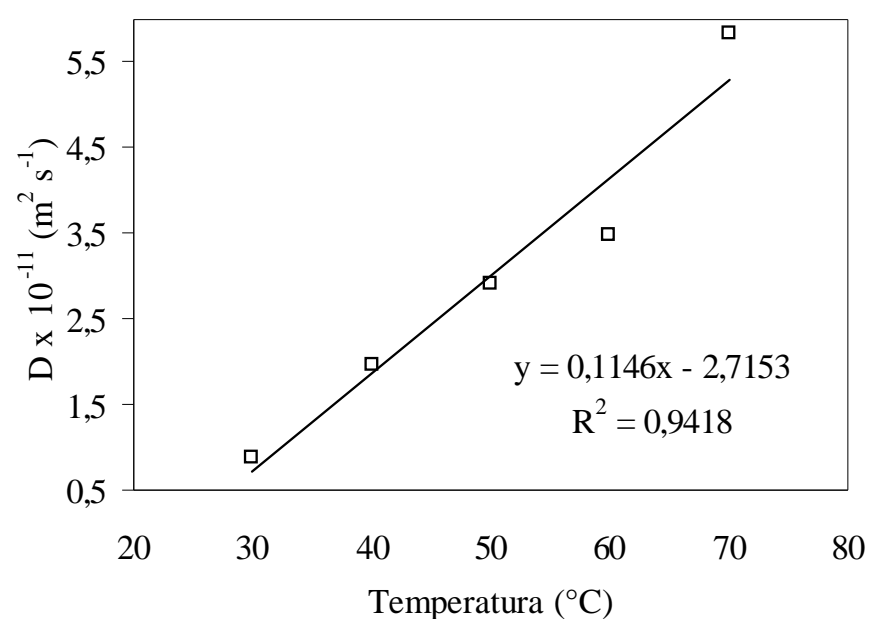

Figura 4. Valores médios do coeficiente de difusão $\left(\mathrm{m}^{2} \mathrm{~s}^{1}\right)$ obtidos para a secagem do Crambe abissynica nas temperaturas de $30,40,50,60$ e $70^{\circ} \mathrm{C}$

o feijão vermelho, que apresentou valores entre $2,1 \times 10^{-10} \mathrm{a}$ $10,4 \times 10^{-10} \mathrm{~m}^{2} \mathrm{~s}^{-1}$ para temperaturas de secagem entre 25 e $55^{\circ} \mathrm{C}$ (Resende et al., 2007). Amendola \& Queiroz (2007) encontraram, durante a secagem de banana a $50^{\circ} \mathrm{C}$, o valor de $4,58 \times 10^{-10}$ $\mathrm{m}^{2} \mathrm{~s}^{-1}$ para o coeficiente de difusão. Babalis \& Belessiotis (2004), relataram magnitudes de $8,40 \times 10^{-10}$ a $1,13 \times 10^{-9} \mathrm{~m}^{2} \mathrm{~s}^{-1}$ para figo quando aumentaram a temperatura de 55 para $85^{\circ} \mathrm{C}$, ou seja, constataram o mesmo com Sharma \& Prassad (2004), com o aumento da temperatura de secagem de alho de 40 para $70^{\circ} \mathrm{C}$, que obtiveram magnitudes de $1,593 \times 10^{-10} \mathrm{e} 9,7 \times 10^{-10} \mathrm{e}$ Sacilik (2007) encontrou magnitudes de $8,53 \times 10^{-11} \mathrm{a} 17,52 \times 10^{-11} \mathrm{~m}^{2} \mathrm{~s}^{-1}$ para o coeficiente de difusão das sementes de abóbora durante a secagem nas temperaturas de 40,50 e $60^{\circ} \mathrm{C}$.

Os valores do coeficiente de difusão efetivo aumentaram linearmente, corroborando com os resultados relatados por Resende et al. (2007); sua dependência com relação à temperatura do ar de secagem foi descrita pela equação de Arrhenius, conforme Figura 5.

A inclinação da curva da representação de Arrhenius fornece a relação $E_{a} / R$, enquanto sua interseção com o eixo das

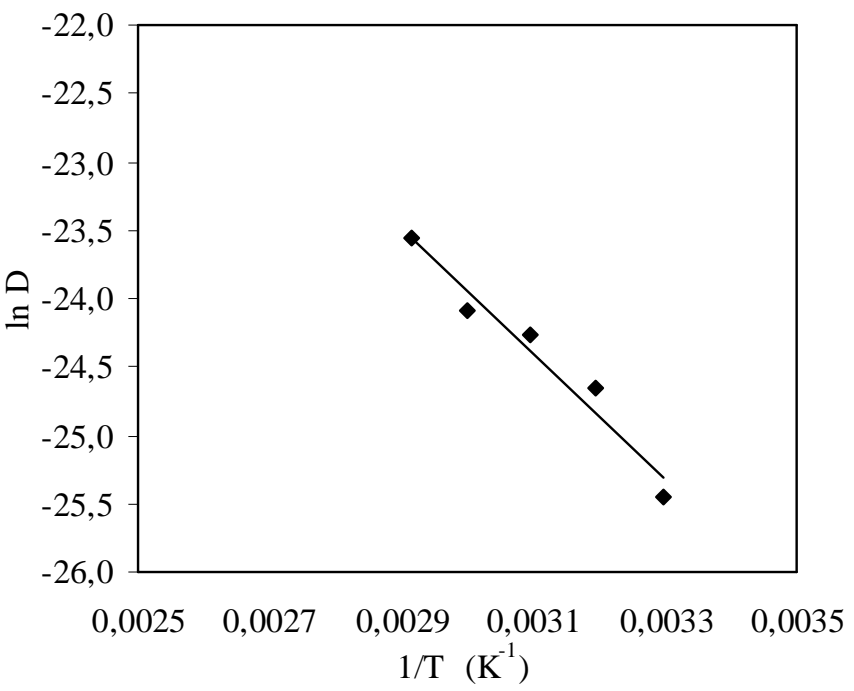

Figura 5. Representação de Arrhenius para o coeficiente de difusão, em função da temperatura do ar de secagem obtido durante a secagem do Crambe abissynica

ordenadas indica o valor de $\mathrm{D}_{\mathrm{o}}$; na Eq. 20 estão apresentados os coeficientes da expressão de Arrhenius ajustada para o coeficiente de difusão efetivo do crambe.

$$
\mathrm{D}=3,45 \cdot 10^{-5} \exp \left(\frac{37.068,26}{\mathrm{R} \cdot \mathrm{T}_{\mathrm{a}}}\right)
$$

Como observado na Eq. 21, a energia de ativação para a difusão líquida no processo de secagem do crambe foi de 37,07 $\mathrm{kJ} \mathrm{mol}^{-1}$. Nos processos de secagem quanto menor a energia de ativação maior será a difusividade de água no produto. Zogzas et al. (1996), ressaltam que a energia de ativação para produtos agrícolas varia entre 12,7 a $110 \mathrm{~kJ} \mathrm{~mol}^{-1}$. A energia de ativação encontrada neste trabalho foi maior que a encontrada para outras oleaginosas, como na mamona, de $15,25 \mathrm{~kJ} \mathrm{~mol}^{-1}$, durante a secagem com uma faixa de temperatura de 25 a $55^{\circ} \mathrm{C}$ (Goneli, 2008) e no nabo forrageiro de $24,78 \mathrm{~kJ} \mathrm{~mol}^{-1}$ para a temperatura de 30 a $70^{\circ} \mathrm{C}$ (Sousa et al., 2011). Goneli et al. (2007), avaliando a secagem de trigo nas temperatura entre $25 \mathrm{e} 55^{\circ} \mathrm{C}$, verificaram que o produto apresentou energia de ativação para a difusão líquida de $42,0 \mathrm{~kJ} \mathrm{~mol}^{-1}$. Reis et al. (2011), secaram pimenta Cumari do Pará para as temperaturas de 45,55 e $65^{\circ} \mathrm{C}$ e obtiveram o valor da energia de ativação de $5,02 \mathrm{~kJ} \mathrm{~mol}^{-1}$. A energia de ativação é uma barreira que deve ser ultrapassada para que o processo de difusão possa ser desencadeado no produto (Kashaninejad et al., 2007). Segundo Kayacier \& Singh (2004), a energia de ativação diminui com a elevação do teor de água inicial do produto durante o processo de secagem.

\section{ConClusões}

1. Com o aumento da temperatura do ar ocorre redução no tempo de secagem das sementes de crambe.

2. Dentre os modelos analisados para representar a secagem das sementes de crambe, o modelo de Wang e Singh foi o que 
melhor se ajustou aos dados nas diferentes condições de secagem.

3. O coeficiente de difusão efetivo aumenta com a elevação da temperatura, durante a secagem do crambe.

4. A relação entre o coeficiente de difusão efetivo e a temperatura de secagem pode ser descrita pela equação de Arrhenius, que apresenta uma energia de ativação de 37,07 kJ $\mathrm{mol}^{-1}$ para a difusão líquida no processo de secagem das sementes de crambe.

\section{Agradecimentos}

Ao CNPq, pela concessão de bolsa ao primeiro autor, e pelo apoio financeiro indispensável na execução do presente trabalho.

\section{LITERATURA CITADA}

Afonso Júnior, P. C.; Corrêa, P. C. Comparação de modelos matemáticos para descrição da cinética de secagem em camada fina de sementes de feijão. Revista Brasileira de Engenharia Agrícola e Ambiental, v.3, p.349-353, 1999.

Akpinar, E. K.; Bicer, Y.; Yildiz, C. Thin layer drying of red pepper. Journal of Food Engineering, v.59, p.99-104, 2003.

Almeida, D. P.; Resende, O.; Costa, L. M.; Mendes, U. C.; Sales, J. F. Cinética de secagem do feijão adzuki (Vigna angularis). Global Science and Technology, v.2, p.72-83. 2009.

Amendola, M.; Queiroz, M. R. de. Mathematical methodologies for calculating the mass diffusion coefficient of bananas during drying. Revista Brasileira de Engenharia Agrícola e Ambiental, v.11, p.623-627, 2007.

Babalis, S. J.; Belessiotis, V. G. Influence of the drying conditions on the drying constants and moisture diffusivity during the thin-layer drying of figs. Journal of Food Engineering, v.65, p.449-458, 2004.

Brasil, Ministério da Agricultura e Reforma Agrária. Secretaria Nacional de Defesa Agropecuária. Regras para análise de sementes. Brasília: Mapa/ACS. 2009. 395p.

Brooker, D. B.; Bakker-Arkema, F. W.; Hall, C. W. Drying and storage of grains and oilseeds. Westport: The AVI Publishing Company. 1992. 450p.

Doymaz, I. Effect of dipping treatment on air drying of plums, Journal of Food Engineering, v.64, p.465-470, 2004.

Ertekin, C.; Yaldiz, O. Drying of eggplant and selection of a suitable thin layer drying model. Journal of Food Engineering, v.63, p.349-359, 2004.

Golz, T. Crambe. Alternative agriculture series. n.4, 1993. http://www.ag.ndsu.edu/pubs/alt-ag/crambe.htm. 2 Jul. 2008.

Goneli, A. L.; Corrêa, P. C.; Resende, O.; Reis Neto, S. A. Estudo da difusão de umidade em grãos de trigo durante a secagem. Ciência e Tecnologia de Alimentos, v.27, p.135-140. 2007.

Goneli, A. L. D. Variação das propriedades físico-mecânicas e da qualidade de mamona (Ricinus communis L.) durante a secagem e o armazenamento. Viçosa: UFV, 2008. 186p. Tese Doutorado
Kashaninejad, M.; Mortazavi, A.; Safekordi, A.; Tabil, L. G. Thin-layer during characteristics and modeling of pistachio nuts. Journal of Food Engineering, v.78, p.98-108, 2007.

Kayacier, A.; Singh, R. K. Application of effetive diffusivity approach for the moisture content prediction of tortilla chips during baking. Lebensmittel-Wissenschaft undTechnologie, v.37, p.275-281, 2004.

Lahsasni, S.; Kouhila, M.; Mahrouz, M.; Jaouhari, J. T. Drying kinetcs of prickly pear fruit (Opuntia ficus indica). Journal of Food Engineering, v.61, p.173-179, 2004.

Madamba, P. S.; Driscoll, R. H.; Buckle, K. A. Thin-layer drying characteristics of garlic slices. Journal of Food Engineering, v.29, p.75-97, 1996.

Martinazzo, A. P.; Corrêa, P. C.; Melo, E. C.; Barbosa, F. F. Difusividade efetiva em folhas de Cymbopogon citratus (DC.) Stapf submetidas à secagem com diferentes comprimentos de corte e temperaturas do ar. Revista Brasileira de Plantas Medicinais, v.9, p.68-72, 2007.

Mohapatra, D.; Rao, P. S. A thin layer drying model of parboiled wheat. Journal of Food Engineering, v.66, p.513-518, 2005.

Moshenin, N. N. Physical properties of plant and animal materials. New York: Gordon and Breach Publishers. 1986. 841p.

Oplinger, E. S.; Oelke, E. A.; Kaminski, A. R.; Putnam, D. H.; Teynor, T. M.; Doll, J. D.; Kelling, K. A.; Durgan, B. R.; Noetzel, D. M. Crambe. Alternative Field Crop Manual, 2000. http://www.hort.purdue.edu/newcrop/afcm/crambe.html. 2 Jul. 2008.

Oliveira, R. A.; Oliveira, W. P.; Park, K. J. Determinação da difusividade efetiva de raiz de chicória. Engenharia Agrícola, v.26, p.181-189, 2006.

Peske, S. T.; Villela, F. Secagem de sementes. In: Peske, S. T.; Rosenthal, M.; Rota, G. R. M. Sementes: Fundamentos científicos e tecnológicos. Pelotas: Editora e Gráfica Universitária(UFPel). 2003. p.283-322.

Reis, R. C.; Barbosa1, L. S.; Lima, M. L.; Reis, J. S.; Devilla, I. A.; Ascheri, D. P. R. Modelagem matemática da secagem da pimenta Cumari do Pará. Revista Brasileira de Engenharia Agrícola e Ambiental, v.15, p.347-353, 2011.

Resende, O.; Arcanjo, R. V.; Siqueira, V. C.; Rodrigues, S. Modelagem matemática para a secagem de clones de café (Coffea canephora Pierre) em terreiro de concreto. Acta Scientiarum, v.31, p.189-196, 2009.

Resende, O.; Corrêa, P. C.; Goneli, A. L. D.; Botelho, F. M.; Rodrigues, S. Modelagem matemática do processo de secagem de duas variedades de feijão (Phaseolus vulgaris L.). Revista Brasileira de Produtos Agroindustriais, v.10, p.1726, 2008.

Resende, O.; Corrêa, P. C.; Jaren, C.; Moure, A. J. Bean moisture diffusivity and drying kinetics: a comparison of the liquid diffusion model when taking into account and neglecting grain shrinkage. Spanish Journal of Agricultural Research, v.5, p.51-58, 2007.

Resende, O.; Rodrigues, S.; Siqueira, V. C.; Arcanjo, R. V. Cinética da secagem de clones de café (Coffea canephora Pierre) em terreiro de chão batido. Acta Amazônica, v.40, p.247-256, 2010. 
Rocha, D. C.; Santos, B. M.; Scheid, C. M.; E Calçada, L. A. Determinação dos coeficientes de difusão e convectivo na Secagem de sementes de nasturtium officinale (agrião). In: Congresso Brasileiro de Engenharia Química em Iniciação Científica, 6, 2005, Campinas. Resumos... Campinas: 2005.

Sacilik, K. Effect of drying methods on thinlayer drying characteristics of hull-less seed pumpkin (Cucurbita pepo L.). Journal of Food Engineering, v.79, p.23-30, 2007.
Sharma, G. P.; Prasad, S. Effetive moisture diffusivity of garlic cloves ander going microwave-convective drying. Journal of Food Engineering, v.65, p.609-617, 2004.

Sousa, K. A.; Resende, O.; Chaves, T. H.; Costa, L. M. Cinética de secagem do nabo forrageiro (Raphanus sativus L.). Revista Ciência Agronômica, v.42, p.883-892, 2011.

Zogzas, N. P.; Maroulis, Z. B.; Marinos-Kouris, D. Moisture diffusivity data compilation in foodstuffs. Drying Technology, v.14, p.2225-2253, 1996. 